\title{
Intermédialités
}

Histoire et théorie des arts, des lettres et des techniques

Intermediality

History and Theory of the Arts, Literature and Technologies

\section{Le ventre du géant}

Mircea Cartarescu et la mnémotechnique

\section{Johanne Villeneuve}

Numéro 1, printemps 2003

Naître

URI : https://id.erudit.org/iderudit/1005447ar

DOI : https://doi.org/10.7202/1005447ar

Aller au sommaire du numéro

Éditeur(s)

Centre de recherche sur l'intermédialité

ISSN

1920-3136 (numérique)

Découvrir la revue

Citer cet article

Villeneuve, J. (2003). Le ventre du géant : Mircea Cartarescu et la mnémotechnique. Intermédialités / Intermediality, (1), 107-122.

https://doi.org/10.7202/1005447ar
Résumé de l'article

Cet article propose une lecture du livre Le rêve de Mircea Cartarescu et des figures qui y entrelacent la mort et la naissance. La poétique tératologique du romancier et poète roumain permet de revenir sur l'héritage de la mnémotechnique ou de "l'art de la mémoire " (Francis Yates) en évoquant la réminiscence, et en liant à la naissance même de la mémoire d'une narratrice l'image de la mort. En cette liaison refont alors surface de vieilles hantises que Cartarescu déforme à dessein : l'écriture devient " vivante », sa matérialité procède à des accouchements monstrueux, et l'expérience de la lecture devient l'expérience paradoxale de la mort. 


\title{
Le ventre du géant: \\ Mircea Cartarescu et la mnémotechnique
}

\author{
J O H A N E V I L L E N E U V E
}

- n 1992 ont été regroupés en traduction française plusieurs récits de l'écrivain

roumain Mircea Cartarescu'. Le rêve ${ }^{2}$ comprend cinq textes d'une souterraine mais indéniable unité (Le joueur de roulette russe, Le jeu, Les gémeaux, Rem, L'architecte) regroupés en trois sections: la première nouvelle est appelée Prologue, la dernière Épilogue, et les trois nouvelles centrales sont regroupées sous le titre La nostalgie. Le livre s'ouvre sur une série de déclarations paradoxales faites par un vieil écrivain «qui n’a plus d'avenir sur terre». Énoncées par un romancier fictif, ces déclarations contredisent ni plus ni moins l'existence du livre que le lecteur est en train de lire. Immédiatement après avoir cité T. S. Eliot en épigraphe, le romancier écrit: «Je note ici ces vers d'Eliot, je ne sais à quelle fin. Ce qui est sûr, c'est qu'ils ne serviront pas d'épigraphe à l'un de mes livres: je n'écrirai plus jamais rien - ce que j'écris maintenant n'est pas de la littérature » (LR, p. 13). Puis:

Quand je serai mort, mon caveau, le recoin où je me trouve, continuera de flotter dans le brouillard compact et noir, menant ces feuillets nulle part afin qu'ils ne soient jamais lus de personne. [...] Mon lecteur en ce moment n'est autre que la mort. Je vois ses yeux noirs, humides, attentifs comme le seraient des yeux de petite fille, lire au fur et à mesure que ces lignes se remplissent. Ce que ces feuillets contiennent, c'est mon plan d'immortalité (LR, p. 14-15).

1. Né en 1956 à Bucarest, Mircea Cartarescu est actuellement assistant à la Chaire de littérature roumaine de l'Université de Bucarest. Il est considéré comme l'un des poètes majeurs de sa génération.

2. Mircea Cartarescu, Le rêve, trad. Hélène Lenz, Paris, Éditions Climats, 1992 [1989]. Désormais, les références à cet ouvrage seront indiquées par le sigle «LR» suivi de la page et placées entre parenthèses dans le corps du texte. 
Le lecteur figure-t-il la mort ou est-ce la mort qui prend la figure du lecteur? L'auteur propose une comparaison surprenante: la «mort lectrice» ou le «lecteur mort» de cette histoire ressemble à une «petite fille». C'est en effet une petite fille qui réapparaîtra plus tard, occupant justement la fonction d'une lectrice dont le regard sur les choses provoque le surgissement d'un passé qui n'est pas le sien, mais qui constitue néanmoins le fruit de son imagination. En lisant «au fur et à mesure que ces lignes se remplissent», la lectrice et l'écrivain se confondent en un unique plan d'immortalité - le vieil auteur accouchant de son personnage et la jeune femme accouchant de l'auteur. Ce que Cartarescu mettra en scène dans la plus longue nouvelle du livre, c'est justement le récit d'une lecture, comme si l'écriture procédait toujours d'abord de la lecture: un narrateur racontera la dernière nuit passée avec son amante, nuit durant laquelle celle-ci lui fait le récit de son enfance, ou plutôt le récit de la mémoire de son enfance. Cette histoire, que Cartarescu appelle Rem, est donc celle d'une petite fille, traversée, marquée de manière indélébile, par l'écriture de la mémoire - une histoire où les inscriptions de la mémoire sont indissociables de la vie, indissociables de ce que la vie contient d'organique et de retours possibles.

Rem renvoie à cette catégorie de la mémoire artificielle appelée reminiscentia. Dans l'histoire racontée par la jeune femme, il est surtout un lieu qu'il faut entendre selon deux registres sémantiques dont la coïncidence poétique fournit au récit sa trame principale: le lieu où l'imagination pénètre, lieu magique où sont thésaurisés les secrets de l'enfance; mais aussi un vieux «dépôt» érigé au milieu d'un champ et à proximité duquel, sous le coup d'une prémonition, une famille de géants a construit une énigmatique et longiligne maison. Le lecteur ne pourra jamais parfaitement distinguer entre ces deux occurrences du Rem. Mais il apprend que, depuis le jour où la protagoniste de cette histoire a connu le Rem, «l'existence réelle [s'est mise à] ressembler aux limbes de la mort» (LR, p. 210). C'est donc le Rem qui fait d'elle une lectrice et qui, du coup, transforme son existence en mémorial: retournant vers son enfance, la narratrice de cette histoire renoue avec le Rem, terme magique par lequel le vivant et le mort forment une seule expressivités. Ce que la mémoire se représente n’est jamais en effet retrouvé intact, épargné par le temps, lavé de toutes ces traces qui, en définitive, témoignent de ce que le passé n’est plus. En revanche, le passé accède à

3. Au sens de Louis Vax: «Le rôle des expressivités étant de nous présenter la face singulière des choses» (Louis Vax, La séduction de l'étrange, Paris, Presses universitaires de France, coll. «Quadrige», 1965, p. 186). 
la mémoire en empruntant les traits d'une évanescente présence. Le mort et le vivant s'y emmêlent.

Cette expressivité, cette singularité par laquelle le vivant et le mort fusionnent, traversent le livre en entier de Cartarescu. La narratrice raconte que deux ans plus tôt, en revoyant une amie d'enfance, elle tenta d'évoquer avec elle, sans succès, le souvenir du Rem. Dans la nuit suivant cette rencontre, elle voit en rêve son amie. Celle-ci est morte et repose dans son cercueil:

Je la contemplais les yeux pleins de larmes quand j’ai observé qu'elle était enceinte. Sous sa robe de dentelle blanche, le ventre bombé paraissait agité de lentes contractions [...] à cet instant précis, le ventre d'Esther s'est brusquement vidé et une forme indéfinie a commencé à nager sous les plis de la robe en se préparant à surgir à la lumière. (LR, p. 214-215)

L'imagination de l'accouchement circule dans le texte entier, faisant glisser divers réseaux métaphoriques en une seule communauté de mouvements: métamorphoses, déploiements, épanouissements. C'est là l'essentiel de la poétique cartarescienne: la figure n'est jamais à prendre comme le signe d'une fermeture, d'un repliement d'un terme sur un autre, mais comme le signe d'un spasme, d'une ouverture sismique où prolifère l'imagination. Dans les autres nouvelles, un corps d'homme se métamorphose lentement sous l'effet des cosmétiques en un corps de femme; ailleurs, un musicien accouche littéralement du cosmos. Ailleurs encore, le narrateur initial revient à la charge : «Lecteur bien aimé, m’astu oublié? [...] Tout ce qui s'est déroulé cette nuit entre nos deux amis... est d'abord passé en fait par mon petit ventre sphérique» (LR, p. 310).

Le Rem, en tant qu'espace corporel procréateur, lieu de gestation et d'incorporation du texte à lire, se précise avec l'histoire des géants qui en habitent le flanc - à moins que ce ne soit le Rem qui habite leurs corps démesurés. Le grandpère, maintenant mort, a œuvré toute sa vie dans un cirque fabuleux où se produisaient des licornes, mais aussi des monstres de toutes sortes. Présenté comme l'homme le plus grand au monde, le géant

exhibait son corps... incroyablement long, tatoué de la nuque aux talons par les plus étranges images qui se puissent voir en rêve. Les encres, les poudres colorées enfoncées dans la peau, piqûre après piqûre, l'avaient transformé en vivante chronique du monde passé, présent et à venir. Ses tatouages paraissaient circuler sous la peau... [...] Le perroquet paré d'un diamant sur le front qui, toutes ailes étirées, ornait aujourd'hui ses omoplates, on le voyait grimper le lendemain jusqu'à son cou et son menton, tandis que le surlendemain, il flottait, fantomatique, au-dessus de [sa tête]. (LR, p. $227-228$ ) 
Le géant est un texte vivant qui impose la fantaisie d'un monde, avec ses paysages, ses jardins, sa faune. On y perçoit les remuements, les afflux, les impulsions de la matière. Si le lecteur du roman est la mort, comme on le prétend dans le prologue, pourquoi se surprendre de ce que l'écriture soit vivante? «Par une soirée d'août... voilà qu'il apparut sur la peau [du géant], travaillée par une jungle de tatouages frénétiques, trois lettres qu'on aurait dites frappées dans du saphir: REM» (LR, p. 228). Ce corps fabuleux, monstre parmi les monstres, grouillante mise en abyme du cirque, puisque le corps en spectacle devient sa propre scène, est donc un lieu et un texte tout à la fois. Les tatouages forment une jungle organique, vivante; et les signes qu'ils dévoilent offrent un spectacle vivant. La lecture se fait ici double monstration : monstration de figures et lecture de monstres.

Le géant est, par définition, immense: rêve de totalité, cosmos, il évoque les âges chaotiques de l'origine et la démesure de la matière. Son pas est lourd et s'enfonce dans la profondeur des choses, mais son regard, portant au loin, est prémonitoire. Il est texte vivant, lieu et figure du Tout. Le petit-fils du géant, géant luimême, offre à la petite fille un coquillage dont l'intérieur est «saturé de centaines de petits sentiers croisés ressemblant à des intestins» (LR, p. 231). Cet objet «corporalisé », dont l'intérieur est « blanc comme un ventre de poisson » (LR, p. 230), doit conduire l'enfant-lectrice au cœur des rêves, sur les sentiers d'une forêt imaginaire menant au Rem, «là-bas», dit-il, où se trouve «le Tout» (LR, p. 244). On comprend qu'aucune mesure, aucune balise ne saurait guider le lecteur et lui permettre de distinguer entre le microcosme de l'objet et le macrocosme de l'univers. Est-ce là le geste auquel est confronté tout lecteur baroque? Tirer de la minuscule figure à lire la totalité possible d'un monde? L'immensité du corps est comprise alors comme l'immensité des voies organiques, merveilleuses et abjectes tout à la fois, menant au Tout, c'est-à-dire à cette rencontre explosive entre l'originel et la mort. Le microcosme et le macrocosme se recouvrent l'un l'autre, tandis que l'œuvre de lecture se poursuit. La dimension cosmique du Rem lui vient de sa puissance de figuration dont le caractère tératologique n'est pas à négliger.

La figure à lire, telle qu'elle apparaît chez Cartarescu, est une force tératologique par laquelle le passé, le mort, prend vie - monstrueusement vie. Retournant à la première nouvelle du livre, on saisit davantage la portée de ce que le vieil auteur indique sur l'écriture qui le lie à un lecteur mort; se moquant de son propre échec littéraire, mais reconnaissant enfin que quelque chose écrit à sa place, que la littérature est «le contraire de la confidence», il avoue:

Dès lors que l'écrivain trace des lignes sur une page, dans la main qui tient le stylo une main étrangère s'insinue comme dans un gant... Tandis que l'image du scripteur, 
se reflétant sur la page, s'échappe en s'éparpillant comme des billes de vif argent où coagulent l'Araignée, le Ver, l'Eunuque, l'Unicorne, ou encore le Dieu - alors même que le scripteur désirait seulement parler de lui-même. La littérature relève de la tératologie. (LR, p. 13)

Loin de conduire à une théorie de l'inspiration, cet aveu d'impuissance est une reconnaissance de ce que la figure poétique, une fois conçue dans sa sphère de lecture, grouille et s'auto-déploie, prolifère comme d'elle-même dans le deuil de l'écrivain. La lecture devient l'acte par lequel s'accomplit le déni de la mort. Car cette autre main, semble nous dire l'auteur, est celle du lecteur - main légère qui permet à l'œuvre de se faire, comme si les choses s'écrivaient toutes seules. Cette «étrangeté » de la main est celle-là même de la mort, celle dont le lecteur est amené ironiquement à faire son deuil. L'autopoïesis n'est garantie qu'à condition que ce lecteur accepte de se mêler à cette mort qui n'a jamais la force de l'expérience mais toujours l'apparence de la figure. Il doit accepter de faire lui aussi comme si les choses «sortaient d'elles-mêmes", comme si elles accouchaient sans souffrance. Il ne s'agit pas de nier la matière et, avec elle, l'animation de la vie. Bien au contraire, si «la pesanteur de toute une vie ${ }^{4}$ » semble s'estomper à la lecture, c'est que la matière se met à jouer sur la gamme des signes, des marques et des figures de l'origine. C'est la douleur même de la vie et de ses accouchements que l'imagination lectrice fait désormais miroiter selon les couleurs et les variations du souvenir, avec la légèreté des choses qui s'estompent. Comme pour la mélancolie, la douleur n'est pas oubliée, elle devient jeu, vertige, griserie.

Le mélancolique est assis au centre d'un monde qu'il est le seul à reconnaître en ses débris éparpillés - petits mobiles éphémères circulant autour de lui, autrement incompréhensibles et vides. Devenu à lui-même son propre monde, le mélancolique s'entoure des ruines de mondes depuis longtemps oubliés. L'air grave, il trône sur l'immortalité et souffre de ne pas mourir ${ }^{5}$. La force de sa gravité attire paradoxalement à lui ces ruines, ces astres éteints qui glissent alors sur

4. Je réfère ici à Maurice Blanchot qui, interrogeant cette légèreté des choses qui semblent s'écrire toutes seules, les voit «sans le sérieux, le travail, les lourdes angoisses, la pesanteur de toute une vie qui s'y est déversée » (Maurice Blanchot, L'espace littéraire, Paris, Éditions Gallimard, 1955, p. 254).

5. Telle est la totale malchance du joueur de roulette russe dont l'histoire est associée par l'écrivain dans son prologue à ces «personnages [qui] ne meurent jamais, [car] ils vivent chaque fois que leur monde est lu» (LR, p. 32). Le joueur, qui gagne toujours à la roulette russe, défie les lois de la probabilité, mais «sa malchance étant totale, il ne pouvait qu'échouer dans son intention de se suicider» (LR P. 33). 
leur orbite en paraissant aussi légers que la poussière. Cette conversion réciproque de la gravité et de la légèreté, voilà à quoi le mélancolique est à l'œuvre bien malgré lui. L'inertie et la concentration génèrent autour de lui le mouvement, la circulation, la révolution. Mais cette collection mouvante de ruines, à la fois hétéroclite et ordonnée, demeure à distance, offrant au regard ses jeux changeants, quelques danses de constellations éphémères.

La tératologie de Cartarescu contient en puissance une telle mélancolie: l'auteur semble naître de sa propre mort, à moins qu'il n'agonise comme de toute éternité. Rappelons la thèse formulée par Giorgio Agamben au sujet de l'invention de l'écriture chez Victor Segalen : l'apparition de l'écriture consacre la dette de la littérature à l'égard de l'origine; car c'est à l'effacement de la performance et de la présence orale qu'invite l'écriture ${ }^{6}$, rendant nécessaire la légitimation $1 \mathbf{2}$ d'un point d'origine. Ironiquement, donc, c'est au moment où le présent de l'origine se perd qu'apparaît la nécessité de la figure de l'auteur qui naît ainsi de son propre retrait. Ce moment de légitimité marque l'instant, redoutable autant que monstrueux (au sens évoqué plus haut), de l’intermédialité dont dépend nécessairement l'invention de l'écriture. C'est en effet l'oralité qui «parle» encore l'aspect tautologique étant ici constituant - au cour même du mutisme de l'auteur et de sa figuration mélancolique.

\section{MÉMOIRE ET FIGURES}

La dimension de la mémoire chez Cartarescu se rattache à ce moment d'intermédialité, de tension et d'ironisation. Pour en saisir toute la valeur, sans doute faut-il revenir sur les intrications de ce que d'aucuns ont appelé «l'art de la mémoire» ou la «mnémotechnique». Dans son célèbre ouvrage, Frances Yates préférait parler d'un «art de mémoire» plutôt que de la mnémotechnique, distinguant du coup l'époque de Cicéron d'une culture plus tardive, soit celle de l'alphabétisation ${ }^{7}$. Se basant sur le Ad Herennium, Yates rappelle combien l'art de mémoire dépend d'une interprétation de l'espace, de règles d'emplacements et de règles d'image; elle confère ainsi à la visualité une importance très grande, non sans montrer implicitement que le passage d'un régime oral à un régime d'écriture se double d'une conception trouble de la mémoire. Pour s'édifier selon

6. Voir Giorgio Agamben, «L'origine de l'oubli. Parole du mythe et parole de la littérature », dans Image et mémoire, Paris, Hoëbeke, 1998 [1983].

7. En anglais, «a culture of literacy». Voir Frances Yates, The Art of Memory, London, Routledge Press, 1966. 
son art, celle-ci doit en effet figurer l'espace, disposer des signes comme autant de rappels intériorisés par l'esprit: «For the places are very much like wax tablets or papyrus, the images like the letters, the arrangement and disposition of the images

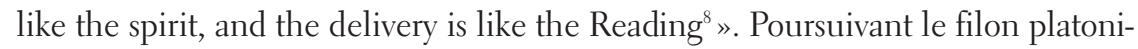
cien (non sans déformation, ni bouleversement, comme nous le verrons bientôt), la mémoire artificielle expose le caractère intermédiatique d'une technique composant avec des figures qui sont à la fois des signes dans l'espace et des expressivités liées au corps et à ses surgissements.

L'histoire de la mnémotechnique commencerait autour de cadavres et de restes. Dans De l'orateur, Cicéron raconte que, soupant un jour chez Scopas, Simonide y chante une ode devant des invités. Prié de sortir, il quitte son hôte avant que ne se produise une chose aussi terrible qu'inattendue:

la salle où Scopas était à table s'écroula, et cette ruine l'écrasa, lui et ses proches.

Comme les parents des victimes qui désiraient ensevelir leurs morts ne pouvaient reconnaître les cadavres affreusement broyés, Simonide, en se rappelant la place que les convives avaient tous occupés sur les lits, permit aux familles de retrouver et d'inhumer les restes de chacun d'eux'.

Simonide fait la démonstration de ce que les choses dont la mémoire cherche à se rappeler s'ordonnent préférablement dans l'espace, selon des images choisies. En cette salle où les convives sont rassemblés chez Scopas naît donc une relation particulière entre la mémoire, le lieu et les images - un lieu à refigurer, un mémorial, puisqu'il s'agit bien d'assurer la sépulture des corps dont la nécessité n'est toutefois plus fondée sur l'unique tradition mémorielle d'une communauté dévouée à ses morts; la mémoire n'est plus l'unique affaire du temps et de la reconnaissance des filiations, des transmissions générationnelles, elle est aussi l'affaire d'un lieu où les cadavres ne sont déjà plus reconnaissables. À l'impératif des honneurs et de l'affection d'une communauté pour ses morts, s'ajoute la nécessité de lire le lieu où les corps, défigurés par la catastrophe, ont perdu le lien qui les unissait à la mémoire de la Cité. Ce passage vers un impératif du lieu témoigne d'un renversement significatif: le passage d'une mémoire encore largement tributaire d'une tradition orale (dont l'ode chantée par Simonide est encore la manifestation coutumière) à une lecture des lieux dont seule une écriture

8. Ad Herennium, cité par Frances Yates, The Art of Memory, p. 7.

9. Cicéron, De l'orateur, trad. Edmond Courbaud, Paris, Éditions Les belles lettres, 1927, Livre II, LXXXVI, p. 351-353. 
peut être porteuse: les lieux, affirme encore Cicéron, «sont les tablettes de cire sur lesquelles on écrit; les images sont les lettres qu'on y trace ${ }^{10} »$.

L'histoire de la mémoire artificielle inaugurée par Simonide est donc celle de l'effondrement d'un lieu, une histoire de cadavres, de corps mélangés et méconnaissables. Basée sur le témoignage oculaire, cette systématisation mnémonique exige un effort de redistribution des images en un lieu transformé en texte, puisqu'il faut bien relier les corps aux restes et en lire la figuration : les corps défigurés reprennent figures. Bien qu'invité à chanter l'ode au banquet, le Simonide de Cicéron s'impose un acte de lecture; pour assurer la mémoire des morts, il n'a pas recours au récit des familles et des actions des hommes comme la tradition orale le prescrit, mais à sa propre présence à titre de témoin oculaire et, surtout, à son habileté à disposer des signes. C'est dans la mesure où il fut présent au souper de Scopas, ancré dans ce lieu, qu'il est en mesure de se confronter à l'irréductible perte que constitue la mort de son hôte et de ses invités. Certes la prégnance de la «vision », le « j’ai vu » se lie au lieu et au souvenir d'un discours et d'un corps tenus là au milieu des convives. En d'autres termes, le témoignage oculaire se lie, à même le manque, à la performance orale, à sa présence ${ }^{11}$. Et voilà comment la réminiscence rend possible l'identification des corps et assure par là même leur sépulture: par la vision tenue et maintenue depuis l'événement du souper jusqu'à la scène des cadavres défigurés - poids sans noms, corps livrés à l'anonyme fracas de la matière. Mais tandis que l'accomplissement de la memoria collective exige cohésion et répétition des rites funèbres, la tâche qui incombe à la mnémotechnique impose une audace; elle exige une tension, une sortie hors de soi, comme en un temps devenu soudainement neutre, bien qu'elle impose l'exercice d'un retour et d'un maintien sur une scène qui n'existe plus, un acte

10. Cicéron, De l'orateur, II, LXXXVI, p. 354.

11. On n'insistera jamais assez sur l'importance de l'acte performatif qu'implique la transmission orale, le but de l'orateur étant d'ancrer son discours dans le monde pratique, dans le monde de l'action (voir Walter J. Ong, Orality and Literacy, Londres, New York, Methuen, 1983). Comme la voix humaine et le son, le discours doit avoir une portée immédiate; le savoir oral est dynamique, dépendant de la force et de la présence d'un corps. C'est bien ce qu'Auerbach reconnaissait chez Homère, quoiqu'il y entendît une carence, soit cette absence d'arrière-plan caractéristique de son épopée («La cicatrice d’Ulysse», Mimesis, trad. Cornelius Heim, Paris, Éditions Gallimard, coll. «Tel», 1968 [1946], p. 1134). Ce que raconte l'épopée homérique constitue toujours le présent, non pas au sens où le passé est occulté, mais au sens où il est toujours là dans la geste collective et dans la voix, remplissant la scène où la performance a lieu. 
de concentration intense. Simonide doit retenir le banquet et son ordonnance spatiale sur le lieu où gisent les cadavres — retenir le vivant dans le mort par l'effort de l'imagination et de son ordonnancement spatial qui le pousse à sortir de soi tout en se maintenant dans l'état d'une totale absorption. L'effort, la tâche, cette sortie qu'accomplit Simonide afin de refigurer ce que la catastrophe a défiguré tient de l'inoui. L'orateur ne trouve en effet aucun secours dans ce qui a été dit ou entendu; la reconstitution, même si elle implique sa propre posture d'orateur, repose sur l'intensité de la présence de son corps dans un lieu qui n'est déjà plus le même, et l'expérience de celui qui a vu. C'est parce qu'il est lui-même sorti de chez Scopas qu'il permet au lieu de sortir à son tour comme hors de soi, afin de mieux revenir en-soi sous la forme de la figuration.

Ainsi, le traité de Cicéron renverse les modalités propres à l'oralité pour concevoir une mémoire en tant que lecture. Si l'oralité pose l'acte de mémoire dans le contexte performatif d'une présence de l'orateur capable de faire parler les Anciens, la mémoire en tant que lecture n'est toujours que le souvenir d'une présence qui, dans le déchirement ou l'écart du temps (ou de la catastrophe), fige sous l'aspect de la figure l'acte performatif lui-même, en révélant le mouvement, la métamorphose, la renaissance du passé. L’orateur se voit ici lié à la technique et à la matérialité de l'écriture: «The art of memory is like an inner writing ${ }^{12}$.» Comme en convient Frances Yates, la technique inaugurée par Simonide dans le traité de Cicéron s'appuie fondamentalement sur le sens de la vue qu'elle place bien au-dessus des autres. Plaçant toute sa confiance dans l'ordonnance de lieux (loci) et d'images faciles à retenir, Cicéron puiserait aisément dans la riche architecture de Rome pour définir les figures de la mnémotechnique et penser leur efficacité. Des lieux et des images ayant un ordre facile déterminé prennent donc figure de ce qu'il y a à rappeler, retenus en mémoire à la place de ce qui n'est pas présent selon une ordonnance qui est celle de lieux fréquentés. Est-ce à dire que la vue se substitue entièrement à l'ouïe dans l'art de mémoire? Certes, c'est bien à une certaine forme de distanciation, voire d'ek-stase, qu'invite la mnémotechnique lorsqu'elle s'appuie sur la vue - contrairement à l'oreille, l'œil est un organe capable d'objectiver, de se mobiliser en fonction de la distance. En fait, l'extase qu'implique la mnémotechnique ne trouve à s'accomplir qu'à travers la fusion artificielle de techniques assorties à l'oralité et à l'écriture. En effet, la question de la présence (dans un lieu tenu là) réfère toujours à la dimension performative de l'oralité mise en scène par le banquet; mais telle est bien l'écriture inté-

12. Frances Yates, The Art of Memory, p. 6. 
rieure de la mémoire qui, accommodée par la métaphysique, peut prétendre présentifier la parole et maintenir son être dans la figure. L'écriture est alors «grosse » d'un accomplissement toujours à naître, posant un paradoxe, puisque ce qu'il y a à naître est le passé lui-même. En retour, la technique de l'écriture se trouve dématérialisée, découpée et mise au service d'un art oratoire millénaire.

Il est significatif que l'histoire de la mnémotechnique commence aussi avec une dette: l'ode que chante Simonide chez Scopas en l'honneur de son hôte est la cause de la catastrophe, car constatant que Simonide y fait aussi un hommage à Castor et Pollux, Scopas prévient le poète qu'il ne lui sera payé que la moitié de la somme prévue. Les Dieux pourront toujours payer la part qui leur est consacrée! Au milieu du banquet, un messager invite Simonide à sortir de la maison de Scopas, car deux jeunes hommes l'attendent à l'extérieur. C'est en son absence que la maison de Scopas s'écroule. Celui-ci paye donc de sa vie de ne pas avoir honoré entièrement le discours de Simonide. C'est là le prix pour l'invention de la mnémotechnique: dans l'ordre qui régit l'ode faite à l'hôte, il suffit que la part consacrée aux Dieux ne soit pas respectée pour que s'ouvre, sous la forme d'une dette dont le paiement est la mort, l'espace d'une écriture des images.

La figure de l'accouchement du mort, telle qu'elle se répand chez Cartarescu, ne devient-elle pas par excellence, de manière tout à fait tautologique, la figure de la figure elle-même en tant qu'elle appartient à une écriture d'images toujours en dette envers la scène performative et vivante de l'oralité? Car la dette entretenue par l'écriture à l'égard de l'oralité pèse lourd, on le sait, depuis la métaphysique platonicienne.

Outre l'incontournable principe d'association énoncé par Aristote dans ce qui constitue la première théorie de l'imagination ${ }^{13}$, le principe selon lequel les images, pour être retenues par la mémoire, doivent être frappantes, choquantes et étranges, quand ce n'est pas grossières, traverse l'histoire de la mémoire artificielle, comme en fait foi le travail de Frances Yates. Or, dans l'histoire de Simonide, c'est la scène de la catastrophe, frappante s'il en est, qui force le souvenir d'images peu frappantes: les invités attablés au banquet. La scène déformée, offerte à la vue et pourtant muette puisqu'elle enferme les identités des morts dans le secret d'une terrible confusion, appelle une scène tout aussi muette - celle du banquet: celle-

13. Voir Aristote, Traité de l'âme, Paris, Éditions Les belles lettres, 1980, mais surtout, De Memoria et Reminiscentia, De la mémoire et de la réminiscence, dans Parva Naturalia, Paris, Vrin, 1951. 
ci pourrait en effet se refuser au souvenir, le passé et l'avenir pourraient se refuser à elle définitivement. Cette scène retrouvera pourtant la voie du temps et, avec elle, celle de la reconnaissance et de l'identité des familles. Mais elle le fera grâce à la figuration par laquelle Simonide la remémore et l'accouche à nouveau figuration d'un lieu et du corps de Simonide au milieu du banquet.

Du point de vue du médium, la réminiscence fait donc ici le pont entre l'oralité et l'écriture; elle dépend, comme la prestation orale, d'une présence tenue et d'une performance; mais elle ne s'accomplit qu'en disposant des signes dans l'espace, préférant la figure à la transmission, la vision à la parole. Grâce à cette première mnémotechnique, Simonide réinvente toutefois l'écriture, en fait une chose vivante à travers laquelle parlent les morts. Car Cicéron renverse l'argument platonicien pour lequel l'écriture est chose morte et le logos, le langage vivant. En traversant la castastrophe à rebours, Simonide puise le nom des morts dans l'écriture vivante d'une scène où les lettres sont les figures organisées autour du festin.

L’intermédialité à l'œuvre dans l'invention de la mnémotechnique, lorsqu'elle se trouve remise en scène par la poétique, ne livre-t-elle pas à l'évidence son caractère tératologique?

\section{TÉRATOLOGIE}

Parlant de la tératologie chez Jérôme Bosch, Roger Caillois la décrivait comme ce qui n'est pas de l'ordre de l'inhumain, mais d'une « contradiction qui porte sur la nature même de la vie et qui n'obtient rien de moins que paraître abolir momentanément, par un vain, mais troublant prestige, la frontière qui la sépare de la mort ${ }^{14}$. » L'œuf semble ouvrir sur des horizons funestes. Jacques Le Goff rappelait, pour sa part, qu'au contraire du merveilleux musulman, le merveilleux occidental tendrait vers une déshumanisation, vers un «univers de monstres ou de bêtes, vers un univers minéralogique [et] végétal ${ }^{15}$.» Ce merveilleux serait tributaire de la résistance médiévale à la vision anthropomorphique de Dieu. Malgré la contradiction apparente entre Caillois et Le Goff sur la place du facteur humain, il y a dans ces tératologies anciennes appelées tantôt fantastiques,

14. Roger Caillois, «Au cœur du fantastique», Cohérences aventureuses, Paris, Éditions Gallimard, coll. «Idées», 1965.

15. Jacques Le Goff, L’imaginaire médiéval, Paris, Éditions Gallimard, 1985, p. 25. 
tantôt merveilleuses, une préoccupation commune. Bien que dénuée de cette déchirure d'avec le réel qu'implique le fantastique moderne, la tératologie, depuis son arrière-pays médiéval, milite en faveur des puissants vertiges et des constructions paradoxales. Le Goff précise: «c'est peut-être ce qu'il y a de plus inquiétant dans ce merveilleux médiéval, le fait justement que l'on ne s’interroge pas sur sa présence sans couture au sein du quotidien ${ }^{16}$. » Il y a, chez Cartarescu, une tératologie où viennent se toucher justement la version de Caillois et celle de Le Goff.

Le Rem de Cartarescu prend forme grâce aux jeux entrepris par des enfants. D'abord dans la nouvelle intitulée Le jeu où des enfants se regroupent, captivés, pour entendre les théories d'un mentor, un petit garçon appelé le Mendébile et qui guide tous leurs jeux. Ces théories préparent à la suite cosmique du livre: selon elles, le crâne du Mendébile contiendrait un univers où se jouerait en abyme le spectacle d'univers multiples orchestré par des «montreurs de marionnettes s'emboîtant les uns dans les autres à l'infini». La terre est décrite par le Mendébile comme «un animal doté de pensée et de volonté». Une fois mort, suggère encore le jeune théoricien, l'homme se retrouverait à l'intérieur des entrailles d'une mère. La gestation du mort, son retour aux entrailles célébré par les enfants ne produit aucune épouvante; au contraire, Cartarescu introduit la tératologie comme une connaissance initiatique par laquelle se rejoignent, en un seul acte de lecture, le vivant et le mort - ce qui se laisse désigner par ailleurs comme «le Tout».

La nouvelle intitulée Rem repose elle aussi essentiellement sur les jeux auxquels s'adonne cette fois un groupe de petites filles. Le fantastique y réinvente ses aspects médiévaux (mythique et merveilleux). D’abord, par la sacralisation des objets ordinaires, ceux qu'utilisent les petites filles dans leurs jeux quotidiens et auxquels est conférée une fonction magique; puis, par la ritualisation du quotidien et sa transfiguration cosmique. L'imaginaire de Cartarescu ne peut donc être appréhendé en fonction d'une fissure unique et fantastique qui séparerait le réel de l'étrange. L'univers évoqué par le livre ne tient pas non plus d'un merveilleux conventionnel. La fouille archéologique de la mémoire à laquelle la lecture est convoquée induit à la fois un espace magique et l'angoisse de la perte.

16. Jacques Le Goff, L'imaginaire médiéval, p. 26. D’où la parenté avec le merveilleux décrit par Tzvetan Todorov dans son célèbre essai (Introduction à la littérature fantastique, Paris, Éditions du Seuil, coll. «Poétique», 1970). 
Cette perdurance de la magie, non pas sous la forme d'un «aspect» d'une œuvre, mais comme fil conducteur de l'art ${ }^{17}$, nous oblige à nous demander si le fantastique et le merveilleux n'exigent pas de notre part, au fond, davantage qu'un travail de classification générique, mais bien une théorie matérielle de la lecture: une théorie qui reposerait sur la disposition de la vision à l'égard de la figure en tant qu'œuvre, ouvrage. Le terme de «tératologie» permet de penser cette disposition et cette fabrication de figures monstrées, elle place la lecture au cœur de la vision qu'implique l'imagination.

Chez Cartarescu, le lecteur qui refuse de «voir» est un lecteur qui refuse de lire. La lecture tératologique concerne, non la capacité herméneutique d'ordonner les strates des significations et leurs niveaux de sens, ni le régime purement sémiotique du texte, mais une posture qui les subsume et les surmonte, par la voie de la figure: cette posture, par laquelle rivalisent distance et proximité, tient à la puissance de figuration du langage plus qu'à sa fonction signifiante. C'est en ce sens qu'on renoue ici avec l'imagination médiévale.

La figure, nous montre le Rem, est affaire d'intensité; la condensation, la métaphore, la comparaison - toutes opérations clés de l'élaboration poétique frappent la vue, l'intensité de la vue, avant d'ouvrir cet espace analytique qui permet d'en comprendre la genèse. Mais qu'est-ce que frapper la vue? L'œil ne se trouve-t-il pas, par un effet plus proche de l'oralité que de la vision, comme «assourdi », happé brusquement par le rythme de l'immédiateté? Avant d'en saisir l'origine et d'en constater les lettres mortes, la figure irradie avec l'intensité d'un choc: la rencontre indissociable de l'origine et de la mort, de la vie et de

17. Cette fouille est coextensive de l'œuvre. Cartarescu donnerait raison à Starobinski sur le fait que c'est le Tout qui fait l'œuvre, et non une imagination qui s'épanouirait dans un domaine distinct, comme une simple composante de l'œuvre. Starobinski critique ainsi les analyses faites par Poulet et Richard: " pour ces critiques, l'imagination s'épanouit dans un domaine distinct: l'image, le symbole, le mythe, le rêve ou la rêverie [...]. Ils explorent l'imaginaire comme des géologues prospecteraient un minerai précieux inégalement réparti dans le sous-sol - rare ici, abondant ailleurs. Pour eux, l'imaginaire n'est pas coextensif au mouvement de l'œuvre, c'en est une composante » (Jean Starobinski, L'œil vivant II, Paris, Éditions Gallimard, 1970, p. 176). Dans le même esprit, André Breton voyait dans l'art une fonction magique indissociable du travail matériel des choses, de leur germination - le spirituel n'étant, pour lui, rien d'autre que cette autopoïesis constante fournie par la matière, par le monde intrinsèque des choses et des événements (André Breton, «L'art magique », avec le concours de Gérard Legrand, Adam Biro, Paris, Phébus, 1991 [1957].) 
l'écriture, comprise comme un déploiement artistique, et non comme un pur scandale. Du coup, l'écriture n'est plus le médium scandaleux, en proie à la perte et au désastre, suspectée de semer l'illusion.

Le Rem de Cartarescu est cet espace vivant et cosmique où l'imagination de la petite fille et la mémoire de la narratrice adulte viennent se croiser violemment, s'imprimant l'une sur l'autre, produisant un art de mémoire. La petite fille porte alors le drame de sa propre mise à mort et la narratrice y retrouve le rêve des origines - leur monstruosité tient à leur capacité de thésauriser les figures de l'origine et de la mort. Ainsi s'accouchent-elles mutuellement. Et comme tout accouchement, celui-ci a l'expressivité d'une double figuration où se conjoignent le mort et le vivant - arrachement à l'être et désir d'être surgissant à l'évidence. À preuve : la tête de l'enfant qui apparaît, tandis que le corps de la mère subit une violente métamorphose. On ne saurait fixer l'unité du corps qui devient monstrueux. Où commence-t-il et où finit-il ? Combien de têtes faut-il dénombrer?

L'accouchement et la métamorphose appartiennent à l'imagination du teratos (monstre), qui trouble à la manière de l'Unheimlich, soit en reposant sur le choc et l'intensité de la vision: la figure submerge le regard, le dévore. L'inquiétante étrangeté n'aurait, en effet, rien à voir avec la notion de « point de vue» par laquelle la perception se voit contrainte ${ }^{18}$. Devant cette force de monstration, l'imagination est à la fois révulsée et attirée; elle n'est pas en mesure d'évaluer la distance ou la proximité avec laquelle la figure émerge.

Avant même que notre petite fille entende parler du géant, elle avait entrepris de dessiner sur le ciment, avec ses amies, « des dessins à la craie colorée, des marelles compliquées et labyrinthiques dont les unes avaient la forme classique d'un homme aux mains étendues de chaque côté» (LR, p. 211). Le jeu finira, lui aussi, par sceller le vivant et le mort; car les sept petites filles vont bientôt, à travers le jeu de «la reine du jour», découvrir un «tunnel à plusieurs étages dont les marches [descendent] dans les profondeurs de la terre» (LR, p. 239). Le corps dessiné devient le théâtre même de la mémoire. La première à y descendre est une fillette appelée «la Baleine». Or cette pénétration funèbre de la terre est la parfaite inversion d'un accouchement - inversion aussi du thème de Jonas: «... déchirant les guirlandes de papier attachées à son cou, elle [la baleine] péné-

18. Louis Vax, dans La séduction de l'étrange, reprochera d'ailleurs à Freud ce manquement à l'égard de l'Unheimlich, à savoir l'absence de considération pour la sphère même de la perception, une oblitération du point de vue dans le cadre d'un acte de vision qui fonde le sentiment d'inquiétante étrangeté. 
tra toute entière au fond du souterrain. On ne voyait plus que le haut de son crâne vacillant dans l'ombre toujours épaisse». Les autres la suivent dans ce corridor «étrange» où «la lumière au lieu de baisser comme on aurait pu s'y attendre, augmentait, bleuâtre et artificielle, tombant de nulle part» (LR, p. 240).

Bien sûr, il s'agit encore de pénétrer le Rem, puisque les fillettes y trouveront un trésor archéologique: le squelette d'un géant, si grand qu'elles pourront y venir jouer et s'y cacher à leur aise. Cette joyeuse accommodation, qui tient du merveilleux médiéval, rappelle encore combien le lieu est précieux à la faculté de la mémoire. L'héroïne de cette histoire, par l'imagination de laquelle surgissent précisément toutes ces figures, se retrouve à son tour dans le Rem. Comme Simonide revenant au banquet par la force de la réminiscence, la fillette retrouve l'ordre d'un cosmos dans le corps du géant devenu maison.

Mais si le géant est un lieu, une maison, un paysage qu'elles escaladent et visitent, un corps qui ressemble à celui d'un reptile antédiluvien, à un vaisseau ou aux restes d'un homme dévoré par une araignée géante, il paraît aussi «avoir quelque chose de commun avec un enfant encore abrité dans le ventre de sa mère» (LR, p. 241). Cette découverte n'est pas attribuable uniquement aux fillettes, mais aussi à la mémoire d'une femme: la fillette devenue narratrice. Cette puissante figure de géant, ce monstre archéologique dans le squelette duquel la mémoire d'une femme retrouve son enfance, ses jeux et le trésor merveilleux d'où naît le monde, exige une lecture tératologique: déploiement de figures qui s'auto-génèrent, mais qui sont ordonnées, ne serait-ce que parce qu'elles gravitent toutes autour d'un noyau central : cette volonté orientée si fortement vers le projet de la totalité cosmique.

Si le lecteur de Cartarescu est la mort, c'est parce qu'il ne saurait lire autre chose que des restes en puissance, des ossements vivants, des visages perdus et retrouvés - toutes figures du manque. Sa tâche n'est pas de reconstituer analogiquement les relations entre un matériau discursif et des figures mentales, mais de remembrer archéologiquement une mémoire afin de livrer passage à la puissance de figuration du langage, en l'occurrence ce qu'on pourrait appeler la poétique du Tout ${ }^{19}$.

Il n'est pas de doute que le livre de Cartarescu s'appuie librement sur la tradition de la memoria. Il n'est pas de doute que le Rem évoque explicitement, tout

19. Le projet artistique de Cartarescu est ambitieux. Cette visée de la totalité, il l'exprime déjà, à en croire l'éditeur français du Rêve (en quatrième de couverture), dans un recueil de poésie portant le titre Le tout et publié en 1985 . 
comme les sept petites filles du roman, l'imagination mnémotechnique et son héritage hermétique. Le trésor que constitue le Rem, dans toutes les variétés de sa figuration et de sa gestation, rappelle ces «palais de la mémoire» dont Augustin célèbre, dans les Confessions, les «trésors d'images».

Accoucher de sa propre mort, remembrer un passé mort par l'agencement des figures comme si elles étaient arbres de vie, voilà la tâche paradoxale confiée à l'écriture et pour laquelle un acte de concentration, d'absorption est nécessaire - ce que l'herméneutique n'a cessé d'enseigner. Mais quelque chose demeure cependant de l'arrière-pays médiéval dans l'écriture et son acte de lecture: le sentiment ou le souvenir d'une présence pleine, d'une expressivité que rien ne sépare de la totalité d'un monde et qui s'éveille à nouveau à travers la péripétie dans laquelle entraînent les signes - ces signes qui ne sont déjà plus des signes (ou pas encore), mais des êtres vivants. C'est du fond de cet arrière-pays qu'apparaît encore l'oralité.

La mémoire détient la clef de cet organigramme poétique si puissamment dessiné par Cartarescu. La lecture tératologique fait voir la mémoire à travers la familiarité des lieux et des objets (lieux de la banalité quotidienne d'une Roumanie reconnaissable, transformés néanmoins en collections, en musées personnels), les références à la littérature prestigieuse (celle du romantisme avec son arrière-pays médiéval, mais aussi la magie surréaliste), où se rejoignent les lectures familières et le sentiment d'étrangeté. Le temps y est alors conçu, selon sa définition mythique et merveilleuse, comme ce qui ne se laisse pas saisir mais où l'on peut aisément retourner.

Le paradoxe énoncé au début du livre, et qui consiste en cette auto-dénégation du roman ( «je n’écrirai plus jamais rien »), est assumé à la manière de Borgès ou de Danilo Kis. Il n'entrave en rien le travail de la mémoire. Au contraire, il permet aux lettres inscrites par l'écrivain sur la page, aux premières figures d'encre coagulée de s'épanouir, de déployer leur étrangeté, afin qu'une mémoire prenne corps et tienne lieu. Ce mouvement de la figure minuscule vers le Tout, de l'infime vers le paysage fait voir une densité figurale. Au lieu de s'enchaîner, les figures de mémoire s'épaississent ou s'étalent. Loin de conférer à la lettre morte, l'écriture s'auto-génère donc, mais tel un monstre de vie incapable de se défaire des liens qui l'attachent à son passé. Par le biais d'une mémoire tératologique, figurable, à lire, l'écriture compose avec sa propre mort comme le vivant avec le devenir. N'y a-t-il pas, dans l'écriture poétique de surcroît, comme un refus d'abandonner aux oubliettes l'intensité d'un imaginaire médiéval et d'une poésie orale pour laquelle la mémoire est toujours un corps vivant et partagé? 